\title{
Axiomatic Relation between Thermodynamic and Information-Theoretic Entropies
}

\author{
Mirjam Weilenmann, ${ }^{1,2}$ Lea Kraemer, ${ }^{1}$ Philippe Faist, ${ }^{1,3}$ and Renato Renner ${ }^{1}$ \\ ${ }^{1}$ Institute for Theoretical Physics, ETH Zurich, 8093 Switzerland \\ ${ }^{2}$ Department of Mathematics, University of York, Heslington, York, YO10 5DD, United Kingdom \\ ${ }^{3}$ Institute for Quantum Information and Matter, California Institute of Technology, Pasadena, California 91125, USA
}

(Received 22 September 2015; revised manuscript received 4 November 2016; published 22 December 2016)

Thermodynamic entropy, as defined by Clausius, characterizes macroscopic observations of a system based on phenomenological quantities such as temperature and heat. In contrast, information-theoretic entropy, introduced by Shannon, is a measure of uncertainty. In this Letter, we connect these two notions of entropy, using an axiomatic framework for thermodynamics [E. H. Lieb and J. Yngvason Proc. R. Soc. 469, 20130408 (2013)]. In particular, we obtain a direct relation between the Clausius entropy and the Shannon entropy, or its generalization to quantum systems, the von Neumann entropy. More generally, we find that entropy measures relevant in nonequilibrium thermodynamics correspond to entropies used in one-shot information theory.

DOI: 10.1103/PhysRevLett.117.260601

Entropy plays a central role both in thermodynamics and in information theory. This is remarkable, as the two theories appear to be fundamentally different. Thermodynamics is a phenomenological theory concerned with the description of large physical systems, such as steam engines or fridges. It relies on concepts like work or heat, which are defined in terms of macroscopic observables. Information theory, on the other hand, deals with "knowledge" on a rather abstract level. Like statistical mechanics, it refers to the microscopic states of a system, such as the values of the individual bits stored in a memory device.

Accordingly, the notion of entropy is rather different in the two theories. In thermodynamics, entropy is a function of the macroscopic state of a physical system that describes phenomenologically which processes are possible independently of any microscopic model. Following Clausius, it is conventionally defined in terms of the heat transfer into a system at a given temperature, and it lends its operational significance from the second law [1,2]. In information theory, entropy was originally introduced by Shannon to quantify the information content of data or, equivalently, the uncertainty one has about them [3]. Operationally, it characterizes properties such as the compression length, i.e., the minimum number of bits needed to store the data. Mathematically, the Shannon entropy is a function of the probability distribution of the random variable that models the data. The von Neumann entropy [4] provides a generalization of this concept to the case where information is represented by the state of a quantum-mechanical system.

Published by the American Physical Society under the terms of the Creative Commons Attribution 4.0 International license. Further distribution of this work must maintain attribution to the author(s) and the published article's title, journal citation, and DOI.
The information theoretic entropy is formally equivalent to the entropy function from statistical mechanics. This relation is conceptually justified through Landauer's principle [5-7]. It entails that the loss of information in an erasure operation on a system, and, hence, the decrease of its information-theoretic entropy, is paired with a heat dissipation in the system's environment. Arguments for Landauer's principle start from microscopic considerations, for example, using standard tools from statistical mechanics [8-10], or explicit microscopic models inspired from information theory [11-21]. An alternative view on the conceptual connection of these entropy measures was proposed by Jaynes in terms of his maximum entropy principle [22]. See also Refs. [6,23-26] for a selection of related approaches.

In this Letter, we take a different approach: we show that the information-theoretic entropy results from applying the definition of thermodynamic entropy to quantum resource theories. This connection is not based on a model borrowed from one particular theory, instead it relates the theory of information to that of phenomenological thermodynamics on an axiomatic level.

Our approach relies on a framework by Lieb and Yngvason [27-30], who give a derivation of thermodynamics, and, in particular, of the thermodynamic entropy, based on abstract axioms. These axioms identify basic properties of a thermodynamic system, which we find to be also fulfilled in the context of resource theories. As a consequence, there is also in this context a state function analogous to the thermodynamic entropy. As we show, the state function in question is none else than the information-theoretic entropy itself. This provides a novel connection between the thermodynamic and information-theoretic entropies.

In particular, this connection extends to the min- and maxentropy, "single-shot" generalizations of the von Neumann entropy. These have been introduced to characterize single 
instances of information-theoretic tasks [31,32]. We show that they, too, can be obtained from the same axiomatic approach as thermodynamic entropy. In order to demonstrate this, we consider an extension of Lieb and Yngvason's framework to nonequilibrium states [29], with which these entropy measures are recovered. Our work bears some resemblance to the study of entanglement theory using similar tools [33-35].

The remainder of this Letter is organized as follows. We start with a summary of the Lieb-Yngvason framework for thermodynamics. As a first technical contribution, we show that the framework is applicable to a microscopic description of thermodynamic systems by resorting to a quantum resource theory. We then show that the corresponding entropy measures defined within the framework coincide with information-theoretic entropies (Proposition 1). We subsequently extend these considerations to other thermodynamic quantities such as the Helmholtz free energy (Proposition 2).

Lieb and Yngvason's approach.-The axiomatic framework by Lieb and Yngvason follows a history of developments towards a mathematically rigorous treatment of thermodynamics [27-29,36-45]. Lieb and Yngvason [27-29] consider the set $\Gamma$ of all equilibrium states of a thermodynamic system and equip this space with an order relation, denoted by $\prec$. For $X$ and $Y \in \Gamma, X \prec Y$ means that the state $Y$ is "adiabatically accessible" from the state $X$ "by means of an interaction with some device consisting of some auxiliary system and a weight in such a way that the auxiliary system returns to its initial state at the end of the process, whereas the weight may have risen or fallen" [27]. It is, moreover, assumed that two systems $X, X^{\prime}$ can be composed, denoted by $\left(X, X^{\prime}\right)$, as well as that a system $X$ can be scaled by a factor $\lambda$, denoted by $\lambda X$. The scaling corresponds to considering a fraction $\lambda$ of the substance in $X$.

Provided $\prec$ obeys some natural axioms, Lieb and Yngvason show that there is an essentially unique thermodynamic entropy $S$ that correctly characterizes all possible state transformations, which is given by

$$
\begin{aligned}
S(X) & =\sup \left\{\lambda:\left((1-\lambda) X_{0}, \lambda X_{1}\right) \prec X\right\} \\
& =\inf \left\{\lambda: X \prec\left((1-\lambda) X_{0}, \lambda X_{1}\right)\right\},
\end{aligned}
$$

where $X_{0}$ and $X_{1}$ are two reference states whose choice alter $S$ only by an affine change of scale [46]. Intuitively, if the state $X$ can be reached adiabatically from $X_{0}$, and $X_{1}$ can be attained from $X$, then the entropy $S(X)$ is defined as the optimal $\lambda$ such that the state $X$ can be created from $X_{0}$ and $X_{1}$ combined at a ratio $(1-\lambda): \lambda$ by an adiabatic process. Physically, $S$ corresponds to the usual thermodynamic entropy as defined by Clausius via the heat $\delta Q_{\text {rev }}$ transferred into a system at a given temperature $T$ in a reversible process, $d S=\delta Q_{\text {rev }} / T$.
Lieb and Yngvason have extended this framework to include certain nonequilibrium states [29]. The states of the corresponding extended state space, $\Gamma_{\text {ext }}$, obey weaker axioms than those of $\Gamma$. For instance, they may not be scalable. The entropy $S$ can, in general, not be uniquely extended to $\Gamma_{\text {ext }}$. However, one can bound all monotonic extensions of $S$ to $\Gamma_{\text {ext }}$ from below and above by two quantities $S_{-}$and $S_{+}$[29]. These quantities give necessary criteria as well as sufficient criteria for adiabatic transitions between thermodynamic nonequilibrium states. Here we use instead slightly adapted quantities, defined as

$$
\begin{aligned}
& \tilde{S}_{-}(X)=\sup \left\{\lambda:\left((1-\lambda) X_{0}, \lambda X_{1}\right) \prec X\right\} \\
& \tilde{S}_{+}(X)=\inf \left\{\lambda: X \prec\left((1-\lambda) X_{0}, \lambda X_{1}\right)\right\} .
\end{aligned}
$$

While they are essentially equivalent to $S_{-}$and $S_{+}$, they are the more natural quantities within the context we consider (cf. also Supplemental Material [47] for a detailed analysis).

Information-theoretic entropy measures.-Information theory is concerned with data and their processing. In quantum information theory, which we consider here for generality, data are encoded in quantum systems [61], whose states are described by the density operator formalism. Throughout this Letter, we restrict our attention to finite-dimensional quantum systems. Information is quantified using an information-theoretic entropy measure, most commonly the von Neumann entropy $H(\rho)=-\operatorname{tr}(\rho \log \rho)$. Note that $\log$ denotes the logarithm with respect to base 2 in this Letter. The information-theoretic significance of the von Neumann entropy can be established in various ways, e.g., axiomatically [3,62-65]. Other useful entropy measures are the min and the max entropy. The min entropy is defined as $H_{\min }(\rho)=-\log \|\rho\|_{\infty}$, where $\|\rho\|_{\infty}$ denotes the maximal eigenvalue of $\rho$. Operationally, it describes the amount of randomness that can be extracted deterministically from data in state $\rho[32,66]$. The max entropy is defined as $H_{\max }(\rho)=\log \operatorname{rank} \rho$, and quantifies the number of qubits needed to store data in state $\rho$ [31].

Equilibrium states and order relation in the microscopic picture.-To apply Lieb and Yngvason's framework to a microscopic description of systems, as employed in information theory, we need to formally specify the various ingredients (such as the order relation) that the abstract framework requires. First, we identify the set of "equilibrium states" of an information-bearing quantum system. These are defined as the class of states represented by flat density operators, that is, operators whose nonzero eigenvalues are all equal [16]. They stand out due to their scalability and comparability, like the equilibrium states in the thermodynamic framework. (The term equilibrium follows Lieb and Yngvason's terminology.)

We define the composition of states as their tensor product. This does not exclude the possibility of correlations being established between subsystems, but it merely 
asserts that before any interaction takes place these systems are independent $[15,16]$. For the composition with an ancilla, this can be ensured by choosing an ancilla that has never interacted with the system before or that has been decoupled through other interactions.

The notion of an "adiabatic process," in the sense of Lieb and Yngvason, translates to any combination of the following three quantum operations; the order relation characterizes which states can be transformed to which others via such a process: (i) composition with an extra ancilla system in an equilibrium state; (ii) reversible and energy-preserving interaction of the system and the ancilla with a weight system [67]; (iii) removal of the ancilla system, whose final reduced state must be the same as its initial state.

Note that these operations are independent of the particular model used to describe the weight system. In fact, the weight system should be understood as a representative for any work storage system, which may also be modeled via a potential, as, e.g., in Ref. [68]. It can also be shown that the above operations are equivalent to the socalled noisy operations $[69,70]$, a quantum resource theory that plays a prominent role in information theory. In general, a resource theory is defined by restricting the set of all quantum operations to a subset, the allowed transformations. Given such a set of allowed transformations, the aim of a resource-theoretic analysis is now to characterize which states can be interconverted and which tasks can be achieved with these transformations. In the case of noisy operations, a state transformation from $\rho$ to $\sigma$ is possible if and only if $\rho \prec_{M} \sigma[69,70]$, where $\prec_{M}$ denotes the matrix majorization relation $[71,72]$. (See also Sec. II A of the Supplemental Material [47] for further details on quantum resource theories).

We define scaling a quantum system by $\lambda \in \mathbb{N}$ to mean combining $\lambda$ such systems, which coincides with the composition operation. For $\lambda=2$, for instance, the state $\rho$ is scaled to a state $\rho \otimes \rho$. [73]. We then extend this scaling operation to any $\lambda \in \mathbb{R}_{\geq 0}$, as is explained in Sec. III of the Supplemental Material [47].

Main results.-We apply Lieb and Yngvason's framework to the microscopic model detailed above. We show that Lieb and Yngvason's axioms are fulfilled in these settings, and, hence, the implications of the framework apply. This allows us to derive corresponding entropy measures, which are furthermore unique for equilibrium states. Specifically, we obtain the following statement, the formal derivation of which is based on properties of the matrix majorization relation. The full proofs may be found in Sec. III of the Supplemental Material [47].

Proposition 1.- - Let states be ordered by the relation $\prec_{M}$ as described above, and let the equilibrium states be those with flat spectrum. Then the unique thermodynamic entropy function $S$ for equilibrium states coincides with the von Neumann entropy $H$. Furthermore, the two entropic quantities relevant for nonequilibrium states, $\tilde{S}_{-}$and $\tilde{S}_{+}$, equal $H_{\min }$ and $H_{\max }$, respectively.

In other words, quantum information theory can be viewed as an instance of thermodynamics in the sense of Lieb and Yngvason, and the corresponding thermodynamic entropy is precisely the information-theoretic entropy.

It is natural to consider other, nonadiabatic processes within the same mathematical framework, for instance, scenarios where the system is in contact with additional reservoirs. In case the system interacts with a heat bath, the equilibrium states can be taken to be the thermal states of fixed temperature $T$, corresponding to the temperature of the bath, as also known from the canonical ensemble in statistical mechanics.

In such a setting, the processes on the system of interest are the thermal operations, introduced in Refs. $[11,14,16]$. These consist of (i) composition with an ancillary system in a thermal state relative to the heat bath at temperature $T$; (ii) reversible and energy-conserving transformation of the system and the ancilla; (iii) removal of any subsystem.

These operations have been extensively studied and used to understand and characterize possible thermodynamic operations in information-theoretic terms (see, for instance, Refs. [14,16,20]). As in Refs. [16,20], we restrict our analysis to states of the system that are block diagonal in the energy eigenbasis. This restriction allows us to avoid technical difficulties when dealing with coherent superpositions of energy levels; other works have also studied general transformations beyond this restriction [74-76].

The thermal operations are characterized with the mathematical notion of thermomajorization [16], denoted here by $\prec_{\mathrm{T}}$. This order relation obeys Lieb and Yngvason's axioms, and, as before, we may deduce corresponding thermodynamic entropy measures.

Proposition 2.-For states ordered by means of thermal operations through the relation $\prec_{T}$ the unique function $S$ for thermal states coincides with the Helmholtz free energy $F$, and the two quantities $\tilde{S}_{-}$and $\tilde{S}_{+}$, relevant for nonthermal states, correspond to $F_{\max }$ and $F_{\min }$ from Refs. [16,20], respectively.

The single-shot measures $F_{\min }$ and $F_{\max }$ were introduced in Refs. $[16,20]$ to describe the work needed for the formation of a state as well as to characterize the extractable work. Proposition 2 follows analogously to Proposition 1, but with the thermomajorization relation instead of the (standard) majorization; the former includes an additional transformation known as Gibbs rescaling [16,17,77-79] (cf. Sec. IV of the Supplemental Material [47]).

Scenarios defined relative to other types of reservoirs, such as a particle [21] or an angular momentum reservoir [80,81], yield analogous results. Various settings, along with their corresponding order relation, equilibrium states, and resulting state functions, are summarized in Table I.

Discussion.-We have shown that, with minor adaptations, Lieb and Yngvason's framework is directly applicable to quantum resource theories, allowing us to put 
TABLE I. An overview on the application of the Lieb-Yngvason framework to various scenarios, as specified in the first column. The first two rows summarize the results of Propositions 1 and 2 (cf. Sec. V of the Supplemental Material [47] for details on the other two scenarios).

\begin{tabular}{lcccc}
\hline \hline Setting & \multicolumn{1}{c}{ Processes } & Equilibrium states & $S$ & $\tilde{S}_{-}, \tilde{S}_{+}$ \\
\hline Isolated system & Noisy operations, $\prec_{M}$ & $\sum_{i}(1 / \operatorname{rank} \rho)\left|X_{i}\right\rangle\left\langle X_{i}\right|$ & Entropy $H$ & $H_{\min }, H_{\max }$ \\
$\begin{array}{l}\text { Interaction with a heat bath } \\
\begin{array}{l}\text { Interaction with a heat bath } \\
\text { and a particle reservoir }\end{array}\end{array}$ & $\begin{array}{l}\text { Thermal operations, } \prec_{T} \\
\begin{array}{l}\text { Interaction with an angular } \\
\text { momentum reservoir }\end{array}\end{array}$ & $\sum_{i}\left(e^{-\beta E_{i}} / Z\right)\left|E_{i}\right\rangle\left\langle E_{i}\right|$ & Free Energy $F$ & $F_{\min }, F_{\max }$ \\
\hline \hline
\end{tabular}

thermodynamic and information-theoretic entropy on the same footing (see also Fig. 1).

More generally, our approach points out the formal and conceptual parallels of phenomenological thermodynamics and quantum resource theories. It thus relates the classical thermodynamic description of a system (based on macroscopic properties) to a description in terms of the information (about the microscopic degrees of freedom) held by an observer. This underscores that the description of a physical system, in particular, its characterization with an entropy function, is observer dependent, hence, subjective.

We have justified the use of the majorization relation based on Lieb and Yngvason's adiabatic operations.

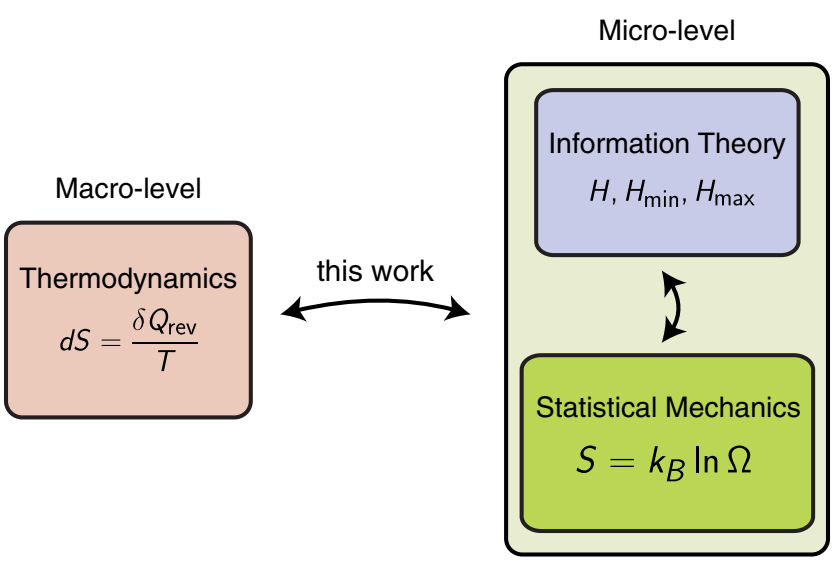

FIG. 1. The contribution of this Letter is to draw a connection between thermodynamic and information-theoretic entropy based on formal axioms and general principles such as additivity and monotonicity that are satisfied within both theories (Proposition 1). This replaces the textbook identification of entropy in thermodynamics and statistical mechanics based merely on analogies, such as common behavior of the two entropy functions in specific models like the ideal gas. The entropy functions in information theory and statistical mechanics are already formally equivalent, and conceptual links have been established through Jaynes' maximum entropy principle [22], as well as through the works of Landauer [5] and Bennett [6] (based on explicit protocols that convert one into the other in an underlying microscopic model).
This relation also occurs naturally in information theory, however. Indeed, it expresses, for instance, an encoding operation or the inverse of a randomness extraction process [82]. We have also shown that order relations characterizing other resource theories are compatible with the axiomatic framework and allow us to derive corresponding "entropy functions" (cf. Proposition 2 and the Supplemental Material [47]). We have in this way recovered the expression of the Helmholtz free-energy as well as corresponding single-shot counterparts $[16,20]$.

For a system interacting with a reservoir, our approach is so far limited to states that are block diagonal in a corresponding eigenbasis, e.g., in the particular case of a heat bath in the energy eigenbasis [16]. We leave for future work the question whether our results also hold for states that do not satisfy this property, which have been studied in Refs. [74-76,83-85].

We expect that the approach presented in this work can be applied to relate other thermodynamic and informationtheoretic quantities. For example, by slightly changing the order relation to a smooth majorization relation, we presume it to yield so-called smooth entropy measures $[31,86]$. Furthermore, we have not treated processes where quantum side information about the system is exploited. This could be useful for performing thermodynamic operations [13]. We might anticipate that an appropriate extension of the Lieb-Yngvason framework would provide an axiomatic and operationally well-justified definition of the conditional entropy.

This project has been supported by the Swiss National Science Foundation (SNSF) via Project Grant No. 200021_153296 and via the NCCR "QSIT," by the European Research Council (ERC) via Project No. 258932, and by the COST Action MP1209.

[1] R. Clausius, Annu. Rev. Phys. Chem. 169, 481 (1854).

[2] R. Clausius, Annu. Rev. Phys. Chem. 201, 353 (1865).

[3] C. E. Shannon, Bell Syst. Tech. J. 27, 379 (1948). 
[4] J. Von Neumann, Dimension Contemporary German Arts And Letters (Springer, New York, 1932), Vol. 42, p. 262.

[5] R. Landauer, IBM J. Res. Dev. 5, 183 (1961).

[6] C. H. Bennett, Int. J. Theor. Phys. 21, 905 (1982).

[7] H. S. Leff and A. F. Rex, Maxwell's Demon 2: Entropy, Classical and Quantum Information, Computing (Taylor \& Francis, 2010, 2010), p. 502.

[8] B. Piechocinska, Phys. Rev. A 61, 062314 (2000).

[9] K. Shizume, Phys. Rev. E 52, 3495 (1995).

[10] D. Reeb and M. M. Wolf, New J. Phys. 16, 103011 (2014).

[11] D. Janzing, P. Wocjan, R. Zeier, R. Geiss, and T. Beth, Int. J. Theor. Phys. 39, 2717 (2000).

[12] O. C. O. Dahlsten, R. Renner, E. Rieper, and V. Vedral, New J. Phys. 13, 053015 (2011).

[13] L. del Rio, J. Åberg, R. Renner, O. Dahlsten, and V. Vedral, Nature (London) 474, 61 (2011).

[14] F. G. S. L. Brandão, M. Horodecki, J. Oppenheim, J. M. Renes, and R. W. Spekkens, Phys. Rev. Lett. 111, 250404 (2013).

[15] J. Åberg, Nat. Commun. 4, 1925 (2013).

[16] M. Horodecki and J. Oppenheim, Nat. Commun. 4, 2059 (2013).

[17] D. Egloff, O. C. O. Dahlsten, R. Renner, and V. Vedral, New J. Phys. 17, 073001 (2015).

[18] P. Faist, F. Dupuis, J. Oppenheim, and R. Renner, Nat. Commun. 6, 7669 (2015).

[19] P. Skrzypczyk, A. J. Short, and S. Popescu, Nat. Commun. 5, 4185 (2014).

[20] F. Brandão, M. Horodecki, N. Ng, J. Oppenheim, and S. Wehner, Proc. Natl. Acad. Sci. U.S.A. 112, 3275 (2015).

[21] N. Yunger Halpern and J. M. Renes, Phys. Rev. E 93, 022126 (2016).

[22] E. T. Jaynes, Phys. Rev. 106, 620 (1957).

[23] J. C. Maxwell, Theory of Heat (Longmans, New York, 1871), p. 312.

[24] M. Tribus and E. McIrvine, Sci. Am. 225, 179 (1971).

[25] G. Lindblad, Commun. Math. Phys. 33, 305 (1973).

[26] A. Wehrl, Rev. Mod. Phys. 50, 221 (1978).

[27] E. H. Lieb and J. Yngvason, Not. Am. Math. Soc. A 45, 571 (1998).

[28] E. H. Lieb and J. Yngvason, Phys. Rep. 310, 1 (1999).

[29] E. H. Lieb and J. Yngvason, Proc. R. Soc. A 469, 20130408 (2013).

[30] E. H. Lieb and J. Yngvason, Proc. R. Soc. A 470, 20140192 (2014).

[31] R. Renner, Ph.D. thesis, ETH Zürich, 2005; arXiv:quant-ph/ 0512258 .

[32] R. König, R. Renner, and C. Schaffner, IEEE Trans. Inf. Theory 55, 4337 (2009).

[33] V. Vedral and E. Kashefi, Phys. Rev. Lett. 89, 037903 (2002).

[34] F. G. S. L. Brandão and M. B. Plenio, Nat. Phys. 4, 873 (2008).

[35] F. G. S. L. Brandão and M. B. Plenio, Commun. Math. Phys. 295, 829 (2010).

[36] C. Carathéodory, Math. Ann. 67, 355 (1909).

[37] R. Giles, Mathematical Foundations of Thermodynamics (Elsevier, New York, 1964).

[38] E. Gyftopoulos and G. Beretta, Thermodynamics: Foundations and Applications (Macmillian, London, 1991).
[39] E. P. Gyftopoulos and G. P. Beretta, Ideal -Gas Mixtures and Solutions (Dover, New York, 2005), Chap. 27, p. 487.

[40] G. P. Beretta and E. Zanchini, in Thermodynamics, edited by M. Tadashi (InTech, 2011), Chap. 2, p. 23.

[41] E. Zanchini and G. Beretta, Entropy 16, 1547 (2014).

[42] G. N. Hatsopoulos and E. P. Gyftopoulos, Found. Phys. 6, 15 (1976).

[43] G. N. Hatsopoulos and E. P. Gyftopoulos, Found. Phys. 6, 127 (1976).

[44] G. N. Hatsopoulos and E. P. Gyftopoulos, Found. Phys. 6, 439 (1976).

[45] G. N. Hatsopoulos and E. P. Gyftopoulos, Found. Phys. 6, 561 (1976).

[46] This refers to a transformation of the form $c_{1} S+c_{0}$ with $c_{1}>0$.

[47] See Supplemental Material at http://link.aps.org/ supplemental/10.1103/PhysRevLett.117.260601 for background material on Lieb and Yngvason's framework (Sec. I) and on quantum resource theories (Sec. II) as well as for technical derivations (Sec. III-V), which includes Refs. [48-60].

[48] M. Horodecki and J. Oppenheim, Int. J. Mod. Phys. B 27, 1345019 (2013).

[49] A. Uhlmann, Wiss. Z. Karl-Marx-Univ. Leipzig, Math. Nat. R. 20, 633 (1971).

[50] A. Chefles, Phys. Rev. A 65, 052314 (2002).

[51] C. B. Mendl and M. M. Wolf, Commun. Math. Phys. 289, 1057 (2009).

[52] M. A. Nielsen, Phys. Rev. Lett. 83, 436 (1999).

[53] M. A. Nielsen and G. Vidal, Quantum Inf. Comput. 1, 76 (2001).

[54] M. A. Nielsen and J. Kempe, Phys. Rev. Lett. 86, 5184 (2001).

[55] R. Horodecki, P. Horodecki, M. Horodecki, and K. Horodecki, Rev. Mod. Phys. 81, 865 (2009).

[56] A. Rényi, Proceedings of the Fourth Berkeley Symposium on Mathematical Statistics and Probability, Vol. 1: Contributions to the Theory of Statistics (University of California Press, Berkeley, California, 1960), p. 547.

[57] M. Tomamichel, Ph.D. thesis, ETH Zurich, 2012, arXiv: 1203.2142.

[58] M. Tomamichel, R. Colbeck, and R. Renner, IEEE Trans. Inf. Theory 55, 5840 (2009).

[59] M. Tomamichel, C. Schaffner, A. Smith, and R. Renner, IEEE Trans. Inf. Theory 57, 5524 (2011).

[60] N. Datta, IEEE Trans. Inf. Theory 55, 2816 (2009).

[61] These include classical systems as a special case.

[62] J. Aczél, B. Forte, and C. T. Ng, Adv. Appl. Probab. 6, 131 (1974).

[63] W. Ochs, Rep. Math. Phys. 8, 109 (1975).

[64] I. Csiszár, Entropy 10, 261 (2008).

[65] B. Baumgartner, Found. Phys. 44, 1107 (2014).

[66] M. Tomamichel, R. Colbeck, and R. Renner, IEEE Trans. Inf. Theory 56, 4674 (2010).

[67] J. Åberg, Phys. Rev. Lett. 113, 150402 (2014).

[68] A. E. Allahverdyan, R. Balian, and T. M. Nieuwenhuizen, Europhys. Lett. 67, 565 (2004).

[69] M. Horodecki, P. Horodecki, and J. Oppenheim, Phys. Rev. A 67, 062104 (2003). 
[70] G. Gour, M. P. Müller, V. Narasimhachar, R. W. Spekkens, and N. Yunger Halpern, Phys. Rep. 583, 1 (2015).

[71] R. Bhatia, Matrix Analysis, Graduate Texts in Mathematics (Springer, New York, 1997).

[72] J. Karamata, Sur une inégalité relative aux fonctions convexes (Publications de'l Institut Mathématique, 1932) Vol. 1, pp. 145-147.

[73] Note that correlations within the system do not affect this scaling operation. The latter corresponds to a scaling from an outside perspective: without splitting the system up and probing it, the knowledge about its internal properties, such as the entanglement of individual particles, is not accessible. Our operations thus avoid the potential issues with entanglement in microscopic systems that were raised in Ref. [30].

[74] K. Korzekwa, M. Lostaglio, J. Oppenheim, and D. Jennings, New J. Phys. 18, 023045 (2016).

[75] M. Lostaglio, D. Jennings, and T. Rudolph, arXiv: 1511.04420.

[76] Y. Guryanova, S. Popescu, A. J. Short, R. Silva, and P. Skrzypczyk, Nat. Commun. 7, 12049 (2016).
[77] E. Ruch, Theor. Chim. Acta 38, 167 (1975).

[78] E. Ruch and A. Mead, Theor. Chim. Acta 41, 95 (1976).

[79] C. A. Mead, J. Chem. Phys. 66, 459 (1977).

[80] S. Barnett and J. Vaccaro, Entropy 15, 4956 (2013).

[81] J. A. Vaccaro and S. M. Barnett, Proc. R. Soc. A 467, 1770 (2011).

[82] By "inverse" we mean the map that inverts the extractor function: It is a stochastic process and thus describes a noisy operation.

[83] M. Lostaglio, D. Jennings, and T. Rudolph, Nat. Commun. 6, 6383 (2015).

[84] A. Winter and D. Yang, Phys. Rev. Lett. 116, 120404 (2016).

[85] T. Baumgratz, M. Cramer, and M. B. Plenio, Phys. Rev. Lett. 113, 140401 (2014).

[86] R. Renner and S. Wolf, Simple and tight bounds for information reconciliation and privacy amplification, Advances in Cryptology-Asiacrypt 2005 (Springer, Berlin, Heidelberg, 2005), p. 199. 